Cite this: Phys. Chem. Chem. Phys., 2014, 16, 6986

Received 10th October 2013, Accepted 27th February 2014

DOI: $10.1039 / c 3 c p 54295 e$

www.rsc.org/pccp

\section{The confined space inside carbon nanotubes can dictate the stereo- and regioselectivity of Diels-Alder reactions $\dagger$}

\author{
Nicole M. Smith, ${ }^{a}$ K. Swaminathan Iyer ${ }^{a}$ and Ben Corry ${ }^{{ }^{b}}$ \\ Chemical reactions inside carbon nanotubes can yield unusual outcomes. Molecular dynamics simulations \\ show that within the confined space of carbon nanotubes, the 1,4-exo adduct of a Diels-Alder cycloaddition \\ may be produced instead of the 9,10-adduct, which is favoured in bulk. The likely product is highly \\ dependent on the nanotube radius and reactant size.
}

The control of chemical reaction pathways at the molecular level presents one of the most important scientific challenges to achieving fully sustainable, thermodynamically efficient chemical processes. ${ }^{1}$ The enormous reaction rate accelerations that enzymes achieve at modest temperatures and their ability to achieve control over reaction selectivity has been an inspiration to design synthetic mimics. ${ }^{2}$ These properties of enzymes are attributed to their highly specific binding of substrates in elaborate pockets, which force the substrates into orientations that favour specific reaction paths. ${ }^{3}$ There are many examples in host-guest chemistry where the inner cavity of supramolecular cavitands have been used to emulate enzymatic pockets to accelerate reactions or gain unusual regioselectivity. ${ }^{4-12}$ Sanders et al., provided an example where the stereochemistry of a DielsAlder reaction could be controlled by confining the transition state inside a cyclic, trimeric porphyrin structure. ${ }^{13}$ It was demonstrated that depending on the structure of the trimer, either the 1,4-exo or the 1,4-endo adduct was obtained. Fujita et al., used a tridendate, triangular ligand with end-capped Pd(II) ions to form a large, hydrophobic cavity for guest binding and subsequent reactions. ${ }^{14,15}$ Inside this cavity, the Diels-Alder reaction between $\mathrm{N}$-cyclohexylmaleimide and 9-hydroxymethylanthracene (also among other anthracenes bearing substituents at the same position) results in adduct formation at the terminal 1,4-position of the anthracene ring. In bulk solution, the same reaction typically results in the formation of an adduct bridging the centre ring (9,10-position) of anthracene, in accordance with the high localization of the $\pi$-electron density at this site.

\footnotetext{
${ }^{a}$ School of Chemistry and Biochemistry, The University of Western Australia, WA 6009, Australia

${ }^{b}$ Research School of Biology, The Australian National University, Acton, ACT 0200, Australia. E-mail: ben.corry@anu.edu.au; Fax: +61 261250313

$\dagger$ Electronic supplementary information (ESI) available: Detailed computaional methods. See DOI: 10.1039/c3cp54295e
}

Another space that could be utilized for controlling stereochemistry is the hollow region inside a carbon nanotube, which has already been demonstrated to be an effective vessel for filling with molecular nanomaterials. Owing to the spatial confinement imparted by the carbon nanotubes, novel and distinct physical and chemical properties are found for the encapsulated materials. ${ }^{16}$ There are many experimental reports of encapsulation of materials such as metallofullerenes ${ }^{17,18}$ and organic molecules ${ }^{19}$ inside single-walled carbon nanotubes (SWCNTs). Chemical transformations have also been observed within this confined space such as the transformations of encapsulated $\mathrm{C}_{60}$ molecules into SWCNTs, formation of metal nanowires using encapsulated metallofullerenes, conversion of encapsulated ferrocene molecules into interior tubes forming double-walled carbon nanotubes (DWNTs), reduction of $\mathrm{Fe}_{2} \mathrm{O}_{3}$ to Fe and the transformation of linear adamantane assemblies. ${ }^{20-24}$

Despite the plethora of research on nanomaterials encapsulated within SWCNTs, there have been almost no studies to illustrate the use of the inner space of SWCNTs to control chemical reactions, with only one modelling study showing how carbon nanopores can influence a decomposition reaction. ${ }^{25}$ The limited number of studies is mainly due to the fact that as-synthesized carbon nanotubes have enormous variations in their diameter and chiral angle, unlike supramolecular assemblies. These physical variations result in striking changes in the space and chemical environment inside the nanotube and in their electronic and optical behaviours. The currently unavoidable structural heterogeneity of as-synthesized SWCNTs prevents their widespread use in many applications. ${ }^{26}$ To provide motivation to overcome this experimental obstacle that prevents the use of SWCNTs to control chemical reactions, here we exploit molecular dynamics simulations to illustrate the potential of using the confined space inside carbon nanotubes to dictate the outcome of chemical reactions.

The Diels-Alder reaction is a fundamental higher-order stereoselective reaction that involves the formation of two 
carbon-carbon bonds. By means of $[4+2]$ cycloadditions, the efficient formation of a cyclohexane skeleton with four successive chiral carbon centres offers synthetic versatility, particularly in the total synthesis of many complex natural products. The endo/ exo-selectivity in the Diels-Alder reaction strongly depends on the substrates, based on Woodward and Hoffmann's frontier molecular orbital interactions and steric interactions between dienes and dienophiles in the [4+2] pericyclic transition states. Therefore, it is quite difficult to control endo/exo-selectivity, since most conventional chiral catalysts cannot discriminate the approach of dienes. In fact, many combinations of dienes with dienophiles, particularly $\alpha, \beta$-unsaturated carbonyl compounds, follow a well-known endo-rule that is based on secondary orbital interactions. However, when steric interactions between dienes and dienophiles overcome these second-order interactions in endotransition states, less-familiar exo-adducts can be predominantly obtained against the endo-rule. Herein, using the Diels-Alder reaction between $\mathrm{N}$-cyclohexylmaleimide/9-anthraldehyde and $\mathrm{N}$-phenylmaleimide/9-anthraldehyde as model systems we show that the diameter of the nanotubes plays a pivotal role in determining the stereochemical outcome of the reaction.

The Diels-Alder reaction of anthracenes in the absence of hosts are known to generally yield the 9,10-adduct, due to the high localization of $\pi$-electron density at the 9,10-position. Indeed, $a b$ initio calculations of the Diels-Alder reactions in the gas phase conducted here showed that out of the three possible products the 9,10 -adduct had a lower energy barrier
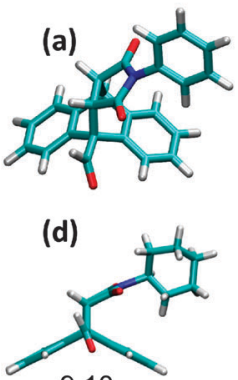

9,10
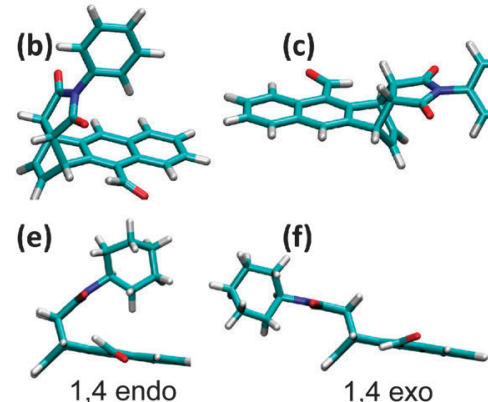

1,4 exo

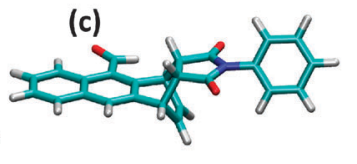

Fig. 1 Optimized structures of possible products from the Diels-Alder reaction between 9 -anthraldehyde with (a)-(c) $N$-phenylmaleimide or (d)-(f) $\mathrm{N}$-cyclohexylmaleimide. 9,10-adducts (a, d), 1,4-endo adducts (b, e) and 1,4-exo adducts (c, f).

Table 1 Gas phase energy barrier to reaction and stability of products with respect to reactants from MP2 geometry optimised ab initio calculations. All values are in $\mathrm{kcal} \mathrm{mol}^{-1 \mathrm{a}}$

\begin{tabular}{llllll}
\hline & (I) & & & \multicolumn{2}{c}{ (II) } \\
\cline { 2 - 3 }$P$ & $E_{a}$ & $\Delta H$ & & $E_{\text {a }}$ & $\Delta H$ \\
\hline 9,10 & 35.6 & -51.5 & & 39.9 & -52.4 \\
1,4 endo & 41.0 & -38.5 & & 46.2 & -39.5 \\
1,4 exo & 50.2 & -32.3 & & 48.8 & -33.2
\end{tabular}

${ }^{a}$ (I) $=N$-phenylmaleimide/9-anthraldehyde; (II) $=N$-cyclohexylmaleimide/9-anthraldehyde; $P=$ product; $E_{\mathrm{a}}=$ activation energy and $\Delta H=$ enthalpy of formation. for formation and greater stability (Fig. 1 and Table 1). The geometries of the optimized products and their transition states differed, with the 9,10-adducts displaying a bent framework and the 1,4-exo and 1,4-endo adducts having the anthracene rings in a planar conformation. The 9,10-adduct with its bent framework may be disfavoured within the confined space of a narrow SWCNT due to steric constraints and reduced $\pi-\pi$ stacking interactions between the anthracene rings and the graphitic SWCNT walls.

Determining how the energetics of the reactions will change within the confines of a SWCNT and accounting for the great number of possible positions of the reactants, SWCNT and solvent molecules is a difficult computational challenge. Rather than directly determining this at an ab initio level, we estimate the potential effect of confinement to the reaction outcomes using molecular dynamics (MD) simulations. While this will never have the level of accuracy for a given conformation as full $a b$ initio results and cannot find transition states, it can yield an idea of how the confined space can limit the approach of the reactants and whether the products can fit in the SWCNT. The reaction activation energy will approximately be modified by any additional energy required to bring the reactants together, and the reaction thermodynamics will be increased by any strain associated with forming the product inside the SWCNT. As classical simulations can sample a large range of conformations, both of these quantities can be estimated in a statistically reliable way to the level of accuracy of the force field. Given that this is difficult to do at an ab initio level we believe that the MD results provide a first indication of what might be expected to occur in the confines of the SWCNT.

The reactants and products of Diels-Alder reactions between $\mathrm{N}$-cyclohexylmaleimide/9-anthraldehyde and $\mathrm{N}$-phenylmaleimide/ 9-anthraldehyde were simulated using $\mathrm{MD}$ in $(8,8)$ and $(9,9)$ SWCNTs with diameters of $10.8 \AA$ and $12.2 \AA$ respectively solvated in benzene. The reactants and products were parameterised using $a b$ initio calculations to be consistent with CHARMM27 force fields. In both cases, when the reactants are unrestrained, the reactive carbons on the maleimide positioned themselves near the 1,4-position of the anthracene ring suggesting that within the confined space of both SWCNTs attack at the 1,4-position is more likely than the 9,10-position (Fig. S1, ESI $\dagger$ ). To understand this further we quantified the energy required to position the reactants in a position suitable for the Diels-Alder reaction to take place. The graphs in Fig. 2 show the required free energy to bring the reacting atoms to the specified separation calculated using umbrella sampling. The energies were compared to the same result found in bulk to show the additional amount of energy required to bring the atoms to the specified separation inside the SWCNTs. For example, in the $(8,8)$ SWCNT, the energy required to position $N$-cyclohexylmaleimide for a reaction leading to a 1,4-exo product (i.e. to bring the reacting carbons to within $4 \AA$ of each other) is very similar to that found in bulk. However, a much greater energy is required to position the reactants for a reaction leading to a 9,10 or 1,4-endo product. On its own, this result does not show which product will form, however when compared to the gas phase calculations 

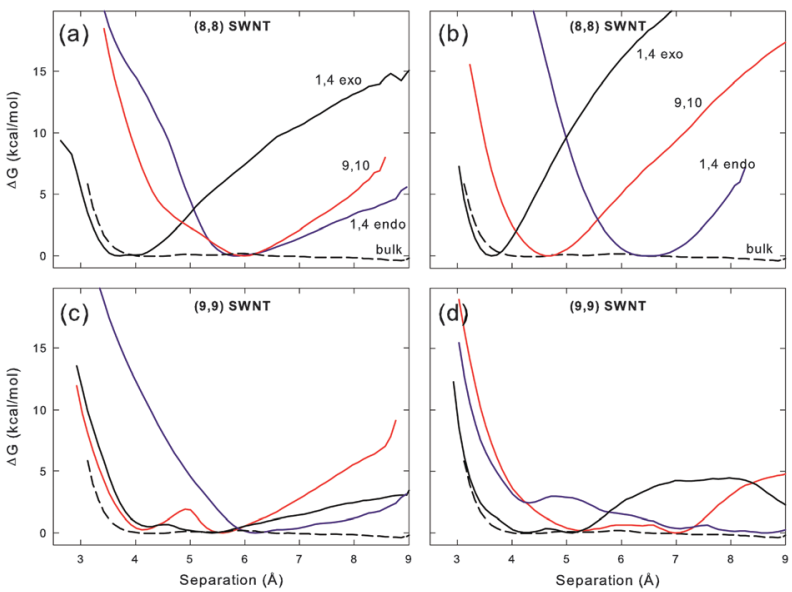

Fig. 2 Free energy profiles (potential of mean force) for reactants for the Diels-Alder reaction of (a, c) N-phenylmaleimide/9-anthraldehyde and (b, d) N-cyclohexylmaleimide/9-anthraldehyde within SWCNTs and in bulk (indicated by dashed line). Profiles were constructed using the method of umbrella sampling.

of the activation barriers for reaction (Table 1) it does give an indication of which product can be expected. The transition states for the reactions have the reacting carbon atoms at a distance of approximately $2.2 \AA$. In the $(8,8)$ SWCNT the additional energy penalty for forming the 9,10 product compared to the 1,4-exo product is greater than $10 \mathrm{kcal} \mathrm{mol}^{-1}$. Comparing this to the ab initio results (Table 1) would suggest that this is sufficient to make the 9,10 product kinetically unfavoured and thus the 1,4-exo product may form. Hence, for the Diels-Alder reactions within the $(8,8)$ SWCNT the likely product is the 1,4-exo adduct irrespective of which dienophile is used. In the case of the $(9,9)$ SWCNT the greater size of $N$-cyclohexylmaleimide makes it harder to find the 9,10 position inside the $(9,9)$ SWCNT than is the case for the $N$-phenylmaleimide. Thus, it may still be possible to form the 1,4-exo product in the larger SWCNT using $N$-cyclohexylmaleimide, but this is unlikely using $\mathrm{N}$-phenylmaleimide with the 9,10-adduct being favoured.

The presence of the constraining SWCNT can also influence the thermodynamic stability of each of the products. Due to the limited space, some products find themselves in a strained configuration in the SWCNT as pictured in Fig. 3, which may also influence their chance of formation. This strain was quantified using molecular dynamics simulations in two ways, in the first case the average internal energy of each product inside the SWCNT compared to that found in bulk solution was calculated during MD simulations. In the second case the free energy change found when moving the product from inside the SWCNT into bulk was calculated. In both cases it is found that the 1,4-exo product is not in a strained configuration in either SWCNT, the 1,4-endo conformation is strained in both cases and the 9,10 conformation is strained in the narrower SWCNT, but not in the wider SWCNT (Fig. 3).

To reinforce the conclusion that the confining SWCNT can influence the thermodynamic stability of each of the reaction outcomes, we quantified its effect on the enthalpy of formation of each of the products using ab initio calculations. To do this,
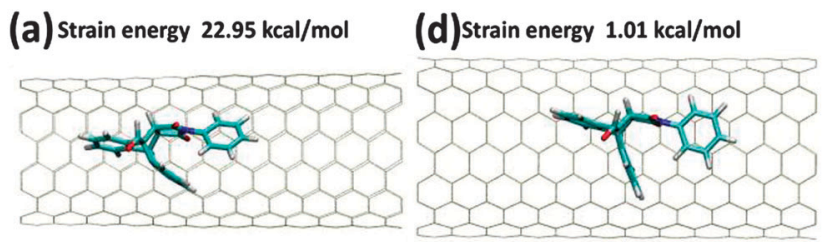

(b) strain energy $46.36 \mathrm{kcal} / \mathrm{mol}$

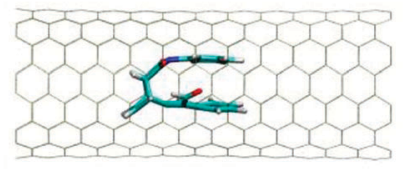

(c) Strain energy $4.02 \mathrm{kcal} / \mathrm{mol}$

(e) Strain energy $37.37 \mathrm{kcal} / \mathrm{mol}$

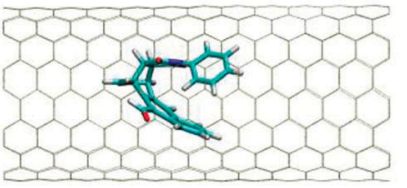

(f) Strain energy $1.98 \mathrm{kcal} / \mathrm{mol}$
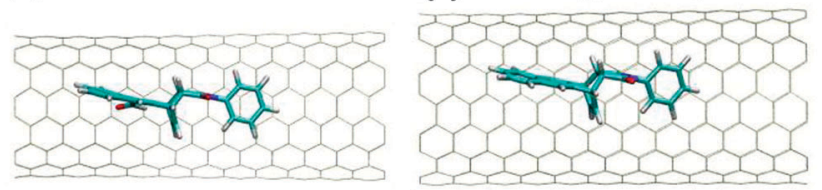

Fig. 3 Energy costs to move products of the $N$-phenylmaleimide/ 9 -anthraldehyde Diels-Alder reaction into $(a-c)(8,8)$ SWCNTs and $(d-f)(9,9)$ SWCNTs from MD. 9,10-adducts ( $a, d), 1,4$-endo adducts (b, e) and 1,4-exo adducts (c, f), found from MD simulations (see ESI† for modelling details).

Table 2 Enthalpies of formation relative to that of the 1,4 exo product found from ab initio simulations for the reaction of $\mathrm{N}$-phenylmaleimide/ 9-anthraldehyde

\begin{tabular}{lrcc}
\hline Product & Gas & $(8,8)$ SWCNT & $(9,9)$ SWCNT \\
\hline 9,10 & -19.2 & -2.7 & -19.1 \\
1,4 endo & -6.2 & 48.0 & 34.2 \\
1,4 exo & 0.0 & 0.0 & 0.0
\end{tabular}

we first found the most likely configuration of the product in the SWCNT using simulated annealing MD simulations. These geometries were then used to determine the $a b$ initio energy of each product, and the resulting relative stability of each product in each SWCNT is shown in Table 2. As was seen from the molecular dynamics results, both the 9,10 and 1,4 endo products are in strained conformations inside the $(8,8)$ SWCNT and thus their thermodynamic stability is reduced compared to the gas phase. In contrast to the gas phase, there is little difference in the enthalpy of formation of the 1,4 exo and 9,10 products in the narrow SWCNT, although the 9,10 product remains favoured in the wider $(9,9)$ SWCNT. Due to the large number of atoms and potential configurations it is not possible to determine the geometry of the transition states inside the SWCNTs so we cannot use ab initio methods to find how the activation barriers are altered inside the SWCNTs. However, as noted above, our MD results suggest a significant increase in the activation barriers for forming the 1,4 endo and 9,10 products inside the $(8,8)$ SWCNT (Fig. 2).

\section{Conclusions}

We have demonstrated using MD simulations that the confined space of SWCNTs may influence both the kinetics and thermodynamics of the Diels-Alder reactions between anthracenes and 
$\mathrm{N}$-substituted maleimide guests, favouring an unusual regioselectivity. If the reaction takes place within the narrower $(8,8)$ SWCNT, formation of the less familiar 1,4-exo product appears to be favoured irrespective of which maleimide dieneophile is used. However, when the reactions are performed in the wider $(9,9)$ SWCNT, the regio-selectivity is influenced by the dieneophile and not just the diameter of the SWCNT. In the $(9,9)$ SWCNT, the 9,10-adduct is favoured when the $\mathrm{N}$-phenylmaleimide dieneophile is used, but when the $\mathrm{N}$-cyclohexylmaleimide dieneophile is used, it may still be possible to form the 1,4-exo adduct due to the greater size of the cyclohexane ring making it harder to find the 9,10 position. In this study we have only examined achiral $(8,8)$ and $(9,9)$ SWCNTs. It is possible that the nanotube chirality could influence the outcome of the reaction as this could alter the preferred orientation of the reactants inside the SWCNT. However, we suspect that the diameter of the SWCNT is likely to have by far the largest influence on the reaction outcome as this will have the biggest influence on how close the reactants can approach each other.

\section{Notes and references}

1 L. Özkan, T. Backx, T. Van Gerven and A. I. Stankiewicz, Chem. Eng. Process., 2012, 51, 109-116.

2 W. P. Jencks, Catalysis in Chemistry and Enzymology, McGraw-Hill, New York, 1969.

3 S. J. Benkovic and S. Hammes-Schiffer, Science, 2003, 301, 1196-1202.

4 J. Chen and J. Rebek Jr, Org. Lett., 2002, 4, 327-329.

5 J. Kang and J. Rebek Jr, Nature, 1997, 385, 50-55.

6 L. S. Kaanumalle, C. L. D. Gibb, B. C. Gibb and V. Ramamurthy, J. Am. Chem. Soc., 2005, 127, 3674-3675.

7 D. Fielder, R. G. Bergman and K. N. Raymond, Angew. Chem., Int. Ed., 2004, 43, 6748-6751.

8 A. Parthasarathy, L. S. Kaanumalle and V. Ramamurthy, Org. Lett., 2007, 9, 5059-5062.

9 L. J. Barbour, G. William Orr and J. L. Atwood, Nature, 1998, 393, 671-673.
10 C. J. Hastings, M. D. Pluth, R. G. Bergman and K. N. Raymond, J. Am. Chem. Soc., 2010, 132, 6938-6940.

11 B. Breiner and J. R. Nitschke, in Supramolecular Chemistry: From Molecules to Nanomaterials, ed. P. A. Gale and J. W. Steed, Wiley, 2011.

12 M. M. J. Smulders and J. R. Nitschke, Chem. Sci., 2012, 3, 785-788.

13 M. Marty, Z. Clyde-Watson, L. J. Twyman, M. Nakash and J. K. M. Sanders, Chem. Commun., 1998, 2265-2266.

14 M. Yoshizawa, M. Tamura and M. Fujita, Science, 2006, 312, 251-254.

15 Y. Nishioka, T. Yamaguchi, M. Yoshizawa and M. Fujita, J. Am. Chem. Soc., 2007, 129, 7000-7001.

16 Z. Wang, Z. Shi and Z. Gu, Chem.-Asian J., 2010, 5, 1030-1038.

17 B. W. Smith, M. Monthioux and D. E. Luzzi, Nature, 1998, 396, 323-324.

18 Y. Sato, K. Suenaga, S. Okubo, T. Okazakai and S. Iijima, Nano Lett., 2007, 7, 3704-3708.

19 T. Takenobu, T. Takano, M. Shiraishi, Y. Murakami, M. Ata, H. Kataura, Y. Achiba and Y. Iwasa, Nat. Mater., 2003, 2, 683-688.

20 S. Bandow, M. Takizawa, K. Hirahara, M. Yudasaka and S. Iijima, Chem. Phys. Lett., 2001, 337, 48-54.

21 R. Kitaura, N. Iazu, K. Kobayashi and H. Shinohara, Nano Lett., 2008, 8, 693-699.

22 L. H. Guan, Z. J. Shi, M. X. Li and Z. N. Gu, Carbon, 2005, 43, 2780-2785.

23 W. Chen, X. L. Pan and X. H. Bao, J. Am. Chem. Soc., 2007, 129, 7421-7426.

24 J. Zhang, Y. Feng, H. Ishiwata, Y. Miyata, R. Kitaura, J. E. P. Dahl, R. M. K. Carlson, H. Shinohara and D. Tomànek, ACS Nano, 2012, 10, 8674-8683.

25 C. H. Turner, J. K. Brennan, J. K. Johnson and K. E. Gubbins, J. Chem. Phys., 2002, 116, 2138-2148.

26 M. S. Arnold, A. A. Green, J. F. Hulvat, S. I. Stupp and M. C. Hersam, Nat. Nanotechnol., 2006, 1, 60-65. 